\title{
Dynamic behaviour and stabilisation to boost the immune system by complex interaction between tumour cells and vitamins intervention
}

\author{
Sana Abdulkream Alharbi ${ }^{1,2}$ and Azmin Sham Rambely ${ }^{2 *}$ (1)
}

\author{
"Correspondence: asr@ukm.edu.my \\ ${ }^{2}$ Department of Mathematical \\ Sciences, Faculty of Science \& \\ Technology, Universiti Kebangsaan \\ Malaysia, 43600, Bangi, Selangor, \\ Malaysia \\ Full list of author information is \\ available at the end of the article
}

\begin{abstract}
In this paper, we establish and examine a mathematical model that combines the effects of vitamins intervention on strengthening the immune system and its role in suppressing and delaying the growth and division of tumour cells. In order to accomplish this, we propose a tumour-immune-vitamins model (TIVM) governed by ordinary differential equations and comprised of two populations, namely tumour and immune cells. It is presumed that the source of vitamins in TIVM originates from organic foods and beverages, based on the food pyramid. The simulation of TIVM employs the fourth order Runge-Kutta method. It is found from the analysis and simulation results that one of the side effects of weakening the immune system is the possibility of transforming immune cells into immune cancer cells to prevent or delay the growth and division of tumour cells. Evidently, for regular intakes of vitamins, which is projected at $55 \%$ of vitamins per day, the immune system is strengthened, preventing the production of tumour cells.
\end{abstract}

Keywords: Dynamic model; Nonlinear ordinary differential equations; Stability; Numerical simulation; Immune cells; Tumour cells; Vitamins

\section{Introduction}

Cancer is defined as unregulated cell growth and division, exemplified by rapid division and invasion of other cells in the adjacent tissue compared to normal cells that grow and divide as a result of either cell death or the replacement of dead cells. Multiple epidemiological studies have shown that transformations of nutrition, lifestyle, environmental inclinations and other factors are closely linked to high mortality rates of several cancers [1-3]. Additionally, the pioneer genomic researcher Craig Venter remarked, at the Leadership for the Twenty-First Century conference, that "Human biology is far more complicated than we imagine. Everybody speaks about the genes that they received from their ancestors for certain traits. However, these genes have very little impact on life outcome. The body is way complex with the involvement of hundreds of thousands of independent factors. Genes are not determined by fate. Genes provide beneficial information about the

(c) The Author(s) 2020. This article is licensed under a Creative Commons Attribution 4.0 International License, which permits use, sharing, adaptation, distribution and reproduction in any medium or format, as long as you give appropriate credit to the original author(s) and the source, provide a link to the Creative Commons licence, and indicate if changes were made. The images or other third party material in this article are included in the article's Creative Commons licence, unless indicated otherwise in a credit line to the material. If material is not included in the article's Creative Commons licence and your intended use is not permitted by statutory regulation or exceeds the permitted use, you will need to obtain permission directly from the copyright holder. To view a copy of this licence, visit http://creativecommons.org/licenses/by/4.0/. 
increased risk of a disease but they will not, in most situations, assess the exact cause of the disease or the actual occurrence of it. Most biological issues come from the complex interaction between all the proteins and cells that function with environmental factors, not specifically influenced by the genetic code" [4]. The relationship between nutrition (such as the A, D, C, E and B-group vitamins) and immunity has recently received significant attention. It has been shown that these vitamins play a vital role in controlling the activity of the immune system to protect tissues from injury [5-7]. In addition, mathematical models have been used to explain disease behaviour over time, which has improved treatment methods. Since 1994, researchers began to mathematically study the behaviour of cancer. A tumour-immune interaction has been studied using the Michaelis-Menten function $[8,9]$. Mayer and others used ordinary differential equations in the formulation of a basic mathematical model to describe the immune system's response when pathogens, such as tumour cells or viruses, invade the body [10]. Ordinary differential equations (ODEs) [11-13], partial differential equations (PDEs) [14] and delay differential equations (DDEs) [15-17] have been used to suggest cancer models and study the effects of tumour growth on the dynamics of other cells or dynamics of brain diseases. Most of these models have been formulated for the development of new cancer therapies. Several models have been formulated to identify the key risk factors of cancer, such as obesity, hormones and diets, but such factors have mainly remained hidden. In 2012, Mufudza and others developed a mathematical model using ODEs to associate the influence of estrogen on the breast cancer dynamics [11]. Roberto and others also used ODEs in the formulation of an obesity-cancer model, which has been used to associate obesity as a cancer risk factor [18], and discussed the connections between obesity and cancer in tumour response to chemotherapy [19]. In 2019, Alharbi and Rambely proposed a healthy-immune model that illustrates dynamically how the immune system prevents the evolution of abnormal cells into tumours and compares its results with the analytical results of an unhealthyimmune model [20]. This study considers unhealthy diet to be one of the main risk factors for increasing cancer cases. In this research, an unhealthy diet has been regarded as one of the major cancer risk factors. With the emergence of abnormal cells in the tissue, vitamins' potential to boost the response of the immune system is dynamically studied such that numerical simulation of the model suggests that the immune system can be boosted when a person regularly intakes vitamins, simulated by $16 \%$ a day [21]. The tumour-immuneunhealthy diet model (TIUNHDM) and the tumour-immune-vitamins model (TIVM) governed by systems of ordinary differential equations are proposed based on models in $[10,20,21]$. The TIUNHDM and TIVM dynamics contribute towards investigating the effects of changes in the rate of vitamin intake on the dynamics of immune and tumour cells.

The remainder of this paper is arranged as follows. TIUNHDM is presented and analysed in Sects. 2 and 3, respectively. The modification of the dynamic behaviour of TIVM by the intervention of vitamins is presented and analysed in Sects. 4 and 5, respectively. Section 6 presents the numerical simulations of both TIUNHDM and TIVM. This paper is concluded in Sect. 7.

\section{The tumour-immune-unhealthy diet model (TIUNHDM)}

In this section, the tumour-immune-unhealthy diet model (TIUNHDM) is developed based on a system of equations called the immune-unhealthy diet model, as described in [20]. In addition, we established TIUNHDM using the following hypothesis: tumour 
cells start to emerge in the tissue as a result of the immune system's inability to eliminate or suppress the abnormal cells, where the immune system's main function is to prevent the body from developing cancer. Previous studies have shown that some unhealthy diet, such as Western-style diet, can cause cancer development and damage the immune system [22-24]. The tumour-immune-unhealthy diet model (TIUNHDM) governed by ordinary differential equations contains two main populations. The first population is the tumour cells, denoted by $T$. In the condition of a weak immune system, this type of cell can divide rapidly while invading and countering other cells in the same or neighbouring tissues [25]. The behaviour growth of tumour cells is represented by the following ordinary differential equation:

$$
\frac{d T}{d t}=\alpha_{1} T\left(1-\alpha_{2} T\right)-\alpha_{3} T I
$$

The rate of the parameter $\alpha_{1}$ determines the growth limit of tumour cells. The second parameter $\alpha_{2}$ represents the tumour reduction due to the deformed tumour from the body during dietary metabolisation. The third parameter $\alpha_{3}$ is the rate of elimination or suppression of tumour cells due to the immune cell response, assuming that the response of the immune system is weak due to the practice of an unhealthy diet.

One of the main functions of the immune system is to prevent the development of cancer in the body. Several immune cells can be generated due to the presence of tumour in the tissue. These cells are sometimes inefficient in activating elimination of tumour cells or delaying their growth. The behaviour of the immune system response, which is denoted by $I$, is represented by the following ordinary differential equation:

$$
\frac{d I}{d t}=\sigma-\delta I+\frac{\rho I T}{m+T}-\mu I T .
$$

The first parameter $\sigma$ represents a constant source of immune cells that are produced daily in the body. The second parameter $\delta$ represents the natural death rate of immune cell. The Michaelis-Menten term $\frac{\rho I T}{m+T}$ discloses immune cells' ability to eliminate tumour cells [10], so that the presence of tumour cells incites the response of the immune system [11]. The parameter $\rho$ represents the rate of this response, and the threshold rate of the immune system is given by the parameter $m$. Additionally, the rapid division and activity of the tumour cells can suppress the activity of the immune cells. The parameter $\mu$ denotes the rate of suppression of the immune cells.

Therefore, TIUNHDM is expressed by the following system:

$$
\begin{aligned}
& \frac{d T}{d t}=\alpha_{1} T\left(1-\alpha_{2} T\right)-\alpha_{3} T I, \\
& \frac{d I}{d t}=\sigma-\delta I+\frac{\rho I T}{m+T}-\mu I T .
\end{aligned}
$$

\section{Analysis of the tumour-immune-unhealthy diet model (TIUNHDM)}

\subsection{Conditions and positivity solution}

TIUNHDM (1) dynamic system is proposed to evaluate the ability of a weak immune system to dynamically suppress tumour cells. Hence, the variables $T$ and $I$ are positive, while the following parameters $\alpha_{1}, \alpha_{2}, \alpha_{3}, \sigma, \delta, \rho, m, \mu$ are real, positive and at most equal to 
one. The feasible region is given as follows:

$$
\Omega=\left\{(T, I) \in R_{+}^{2}\right\}
$$

The initial values of TIUNHDM (1) are selected as follows:

$$
T(0)=1 \quad[26] \quad \text { and } \quad I(0)=1.22 \quad[20,21] .
$$

For all time $t$, the set solutions of the positive conditions are also positive. Hence, the following theorem is obtained.

Theorem 3.1 For any time $t$, the dynamic system region of TIUNHDM $\Omega \subset R_{+}^{2}$ is positivity-invariant, and there exist positive solutions.

Proof Let

$$
\Omega=\Omega_{c}:=\left\{(T, I) \in R_{+}^{2}, T=\frac{1}{\alpha_{2}}, I=\frac{\sigma}{\delta}\right\}
$$

for any time $t$, the set solution $(T(t), I(t))$ of TIUNHDM (1) is positive. From the first equation of TIUNHDM (1), we get

$$
\frac{d T}{d t} \leq \alpha_{1} T\left(1-\alpha_{2} T\right)
$$

By using Bernoulli's method, the solution of equation (2) will be

$$
T(t) \leq \frac{1}{\alpha_{2}+c e^{-\alpha_{1}}} .
$$

As $t \rightarrow \infty$, we get

$$
T(t) \leq \frac{1}{\alpha_{2}}
$$

We show that the solution of the second equation of TIUNHDM (1) is positive for all time $t$, using a separate variables process, and is given by

$$
I(t)=\frac{\sigma}{\delta} .
$$

\subsection{Equilibruim points}

The steady states of the TIUNHDM dynamic system in (1) occur as follows:

- $\frac{d T}{d t}=0 \Leftrightarrow$

$$
T\left(\alpha_{1}\left(1-\alpha_{2} T\right)-\alpha_{3} I\right)=0 .
$$

- $\frac{d I}{d t}=0 \Leftrightarrow$

$$
\begin{aligned}
& \sigma-\delta I+\frac{\rho I T}{m+T}-\mu I T=0, \\
& (\sigma-\delta I)(m+T)-\mu(m+T) I T+\rho I T=0 .
\end{aligned}
$$


Hence, the equilibrium points of TIUNHDM (1) are given by solving equations (3) and (4):

First, by solving equation (3), for $T$, we get

$$
T=0 \quad \text { or } \quad T=\frac{\alpha_{1}-\alpha_{3} I}{\alpha_{1} \alpha_{2}}
$$

By substituting $T=0$ into equation (4), the following value of $I$ is obtained:

$$
I=\frac{\sigma}{\delta}
$$

Hence, the first equilibrium point is given by

$$
p_{0}=\left(0, \frac{\sigma}{\delta}\right)
$$

By substituting $T=\frac{\alpha_{1}-\alpha_{3} I}{\alpha_{1} \alpha_{2}}$ into equation (4), we get one real solution, while the other solutions will be complex. The real solution is given by

$$
I=\frac{1}{6 \alpha_{3}^{2} \mu}\left[2 \alpha_{2} \alpha_{3} A_{1}+\frac{2^{\frac{4}{3}} \alpha_{1} \alpha_{3}^{2}\left(-\alpha_{1} A_{1}^{2}+B_{1}\right)}{\left(C_{1}+\sqrt{C_{1}^{2}+4 D_{1}}\right)^{\frac{1}{3}}}\right]+\frac{1}{3 * 2^{\frac{1}{3}} \alpha_{3}^{2} \mu}\left(C_{1}+\sqrt{C_{1}^{2}+4 D_{1}}\right)^{\frac{1}{3}},
$$

where

$$
\begin{aligned}
A_{1}= & 2 \mu+\alpha_{2}(\delta+m \mu-\rho)>0, \\
B_{1}= & 3 \mu\left[\alpha_{1}\left(1+m \alpha_{2}\right)\left(\alpha_{2} \delta+\mu\right)-\alpha_{1} \alpha_{2} \rho+\alpha_{2} \alpha_{3} \sigma\right]>0, \\
C_{1}= & \alpha_{1}^{2} \alpha_{3}^{3}\left[\alpha_{1} A_{1}\left(-\mu\left(A_{1}-\mu\right)+\alpha_{2}^{2}\left(2 \delta_{2}^{2}+2(-m \mu+\rho)^{2}-\delta_{2}(5 m \mu+4 \rho)\right)\right)\right] \\
& +9\left(\mu+\alpha_{2}\left(\delta_{2}+2 m \mu+\rho\right)\right) \alpha_{1}^{2} \alpha_{2} \alpha_{3}^{4} \mu \sigma<0
\end{aligned}
$$

and

$$
\begin{aligned}
D_{1} & =-\alpha_{1}^{3} \alpha_{3}^{6}\left[\alpha_{1}\left(\mu\left(A_{1}-\mu\right)+\alpha_{2}^{2}\left(\delta^{2}+(-m \mu+\rho)^{2}-\delta_{2}(m \mu+2 \rho)\right)\right)-3 \alpha_{2} \alpha_{3} \mu \sigma_{2}\right]^{3} \\
& >0 .
\end{aligned}
$$

Furthermore, the second equilibrium point is given by $p_{1}=\left(T_{1}, I_{1}\right)$, where

$$
\begin{aligned}
& T_{1}=\frac{1}{6 \alpha_{2}}\left[6-\frac{2^{\frac{2}{3}}\left(C_{1}+\sqrt{C_{1}^{2}+4 D_{1}}\right)^{\frac{1}{3}}+2 \alpha_{1} \alpha_{3} A_{1}}{\alpha_{1} \alpha_{3} \mu}+\frac{2^{\frac{4}{3}} \alpha_{3}\left(-\alpha_{1} A_{1}^{2}+B_{1}\right)}{\mu\left(C_{1}+\sqrt{C_{1}^{2}+4 D_{1}}\right)^{\frac{1}{3}}}\right], \\
& I_{1}=\frac{1}{6 \alpha_{3}^{2} \mu}\left[2 \alpha_{2} \alpha_{3} A_{1}+\frac{2^{\frac{4}{3}} \alpha_{1} \alpha_{3}^{2}\left(-\alpha_{1} A_{1}^{2}+B_{1}\right)}{\left(C_{1}+\sqrt{C_{1}^{2}+4 D_{1}}\right)^{\frac{1}{3}}}\right]+\frac{1}{3 * 2^{\frac{1}{3}} \alpha_{3}^{2} \mu}\left(C_{1}+\sqrt{C_{1}^{2}+4 D_{1}}\right)^{\frac{1}{3}} .
\end{aligned}
$$

Remark 3.1 By applying Theorem 3.1, we deduce that there are no complex equilibrium points for TIUNHDM (1).

Therefore, biologically, the equilibrium points of TIUNHDM $p_{0}$ and $p_{1}$ are classified as follows: 
- The response stage $p_{0}$ : This stage indicates the immune system's ability to respond when tumour cells start to appear in the tissue.

- The interaction stage $p_{1}$ : The immune cells are activated to be active in the interaction due to the presence and behaviour of tumour cells in the tissue despite the weak immune system.

Remark 3.2 By applying Theorem 3.1 and the biological meaning of the equilibrium point $p_{1}$, it is deduced that:

- The tumour cells compete for survival at the interaction stage by rapidly dividing and growing. Therefore,

$$
1 \leq T_{1}<\frac{1}{\alpha_{2}}, \quad \text { where } 0<\alpha_{2}<1
$$

- The rate of the immune cells active in the interaction will be reduced due to the weakness of the immune system during the interaction stage. Therefore,

$$
1 \leq I_{1}<\frac{\sigma}{\delta}, \quad \text { where } 0<\frac{\sigma}{\delta}=1.22
$$

\subsection{Stability of tumour-immune-unhealthy diet model (TIUNHDM)}

From the concept of the Hartman-Grobman theorem [27], the Jacobian matrix of the nonlinear dynamic system of TIUNHDM (1) is given by

$$
J[T, I]=\left[\begin{array}{ll}
F_{T}[T, I] & F_{I}[T, I] \\
G_{T}[T, I] & G_{I}[T, I]
\end{array}\right],
$$

where $F[T, I]=\frac{d T}{d t}$ and $G[T, I]=\frac{d I}{d t}$. According to the statements above, the stability cases of the equilibrium points of TIUNHDM (1) are as presented in Theorem 3.2.

Theorem 3.2 The response stage equilibrium point $p_{0}$ of TIUNHDM (1) is an unstable point.

Proof For analysing the behaviour of $p_{0}$, Jacobian matrix (5) at $p_{0}$ is given as follows:

$$
J[T, I]_{p_{0}}=\left[\begin{array}{cc}
\alpha_{1}-\frac{\alpha_{3} \sigma}{\delta} & 0 \\
\frac{\alpha_{2}-\mu m}{\delta m} & -\delta
\end{array}\right] .
$$

We have

$$
\operatorname{Det}\left(J[T, I]_{p_{0}}\right)=\left(-\delta\left(\alpha_{1}-\frac{\alpha_{3} \sigma}{\delta}\right)\right)<0,
$$

where the sign of $\left(\alpha_{1}-\frac{\alpha_{3} \sigma}{\delta}\right)$ is affected by the hypothesis of TIUNHDM. If the immune system is weak, the rate of suppression of tumour cells by the immune cells denoted by $\alpha_{3}$ will be small compared to the rate of tumour cells growth. Hence,

$$
\alpha_{1}-\frac{\alpha_{3} \sigma}{\delta}=\alpha_{1}-\alpha_{3} I>0
$$


This indicates that matrix (6) has two eigenvalues, which are real and of opposite sign. This implies that the response stage $p_{0}$ is unstable.

Theorem 3.3 TIUNHDM has a stable interaction equilibrium point $p_{1}$.

Proof For analysing the behaviour of $p_{1}$, Jacobian matrix (5) at $p_{1}$ is given as follows:

$$
J[T, I]_{p_{1}}=\left[\begin{array}{cc}
\alpha_{3} I_{1}-\alpha_{1} & -\alpha_{3} T_{1} \\
I_{1}\left(\frac{m \rho}{\left(m+T_{1}\right)^{2}}-\mu\right) & \frac{\rho T_{1}}{m+T_{1}}-\delta-\mu T_{1}
\end{array}\right] .
$$

Considering remark (3.2), we rewrite Jacobian matrix (7) at $p_{1}$, such that $T_{1}=1$ and $I_{1}=1$, as follows:

$$
J[T, I]_{p_{1}}=\left[\begin{array}{cc}
\alpha_{3}-\alpha_{1} & -\alpha_{3} \\
\frac{m \rho}{(m+1)^{2}}-\mu & \frac{\rho}{m+1}-\delta-\mu
\end{array}\right] .
$$

Now, we compute the characteristic equation of Jacobian matrix (8) as follows:

$$
\lambda^{2}-F_{2} \lambda+H=0
$$

where

$$
\begin{aligned}
& F_{2}=\operatorname{trac}\left(J[T, I]_{p_{1}}\right)=\alpha_{3}+\frac{\rho}{m+1}-\alpha_{1}-\delta-\mu<0, \\
& H=\operatorname{det}\left([J T, I]_{p_{1}}\right)=\left(\alpha_{1}-\alpha_{3}\right)\left[\delta-\frac{\rho}{(m+1)^{2}}\right]+\left(\alpha_{1}-2 \alpha_{3}\right)\left[\mu+\frac{m \rho}{(m+1)^{2}}\right]>0 .
\end{aligned}
$$

Then the Routh-Hurwitz theorem is applied for the characteristic equation (9) as follows:

$$
\left|\begin{array}{ccc}
\lambda^{2} & 1 & H \\
\lambda^{1} & -F_{2} & 0 \\
\lambda^{0} & H & 0
\end{array}\right|
$$

The equilibrium point $p_{1}$ is stable for all time $t$ as indicated by the positivity of the elements in the first column.

An evaluation of the immune-healthy diet model (IHDM) [20] shows that the immune system role is classified as healthy if it succeeds in passing three following stages of elimination or suppression of disease pathogens, namely, stable primary stage, unstable interaction stage and stable recovery stage. The following remark is evident from the analysis of the TIUNHDM dynamics.

\section{Remark 3.3}

- TIUNHDM has an unstable response stage, which implies that the immune system is weak and tumour cells have chances to divide and grow rapidly [25].

- TIUNHDM has a stable interaction stage, which shows that immune cells might transfer to immune cancer [11]. Furthermore, this point mathematically indicates that the number of tumour cells starts to escalate, while the number of immune cells is 
Figure 1 Phase portrait of TIUNHDM and solutions around its equilibrium points

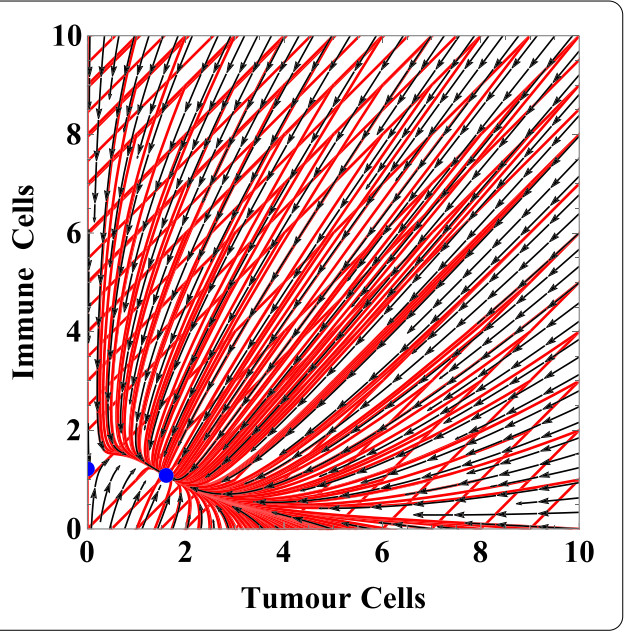

reduced to less than the initial number of immune cells. Figure 1 illustrates the phase portrait of TIUNHDM and its solutions around the equilibrium points.

\section{Modification of the tumour-immune-unhealthy diet model (TIUNHDM) by vitamins intervention (TIVM)}

In 2018, the third report from WCRF and AICR [28] recommended a dietary source of vitamins from natural foods and beverages. However, high doses of dietary supplements is not recommended due to their unforeseen side effects, especially in cancer patients. In 2019, Alharbi and others deduced dynamically that switching to a healthy diet with a rich source of vitamins, estimated at $16 \%$ per day, could boost the immune system and prevent the body from accumulating progressive abnormal cells, such as tumour cells [21]. Based on these recommendations, the behaviour of the dynamic system TIUNHDM was expanded with vitamins intervention derived from a healthy diet pattern as an external factor. In order to achieve this, moderate dietary patterns are suggested following the food pyramid, as shown in $[7,20,26,29]$. This food pyramid follows recommendations from a report from the World Cancer Research Fund (WCRF) and the American Institute for Cancer Research (AICR), which recommends drinking water, eating a diet rich in whole grains, vegetables, fruits and beans, and having a lower intake of red and processed meats, as well as sugars and sweets, as indicated in Fig. 2. Though nutrients and glucose are necessary for cell growth, there are differences between glucose metallisation by cancer cells and other cells [30].

Hence, the tumour-immune-vitamins model (TIVM) is designed to study the effects of vitamins intervention when tumour cells are pose in tissue from stages I and II. The intervention of vitamins is denoted by $V$. It is assumed that a regular rate of vitamins from natural sources of food and beverages (external factor) denoted by $k_{1}$ has been consumed by the individual, and the glucose rate is maintained in the blood. Thus, the rate of vitamins which are attracted by cells is denoted by $k_{2}$. The intervention of vitamins equation is given as follows:

$$
\frac{d V}{d t}=k_{1}-k_{2} V
$$




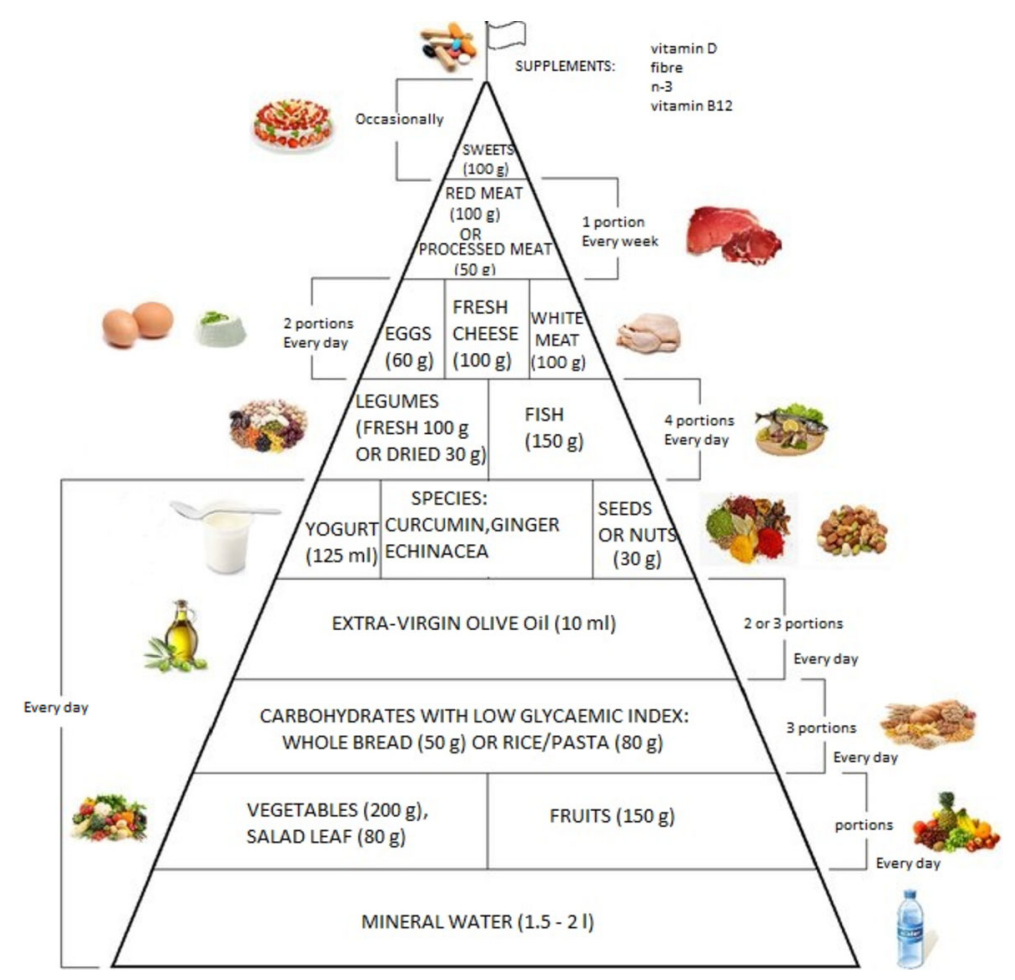

Figure 2 Management of a diet based on the recommendation by WCRF and AICR, where the amounts of food are estimated by nutritional analysis

The vitamins intervention effect on the dynamic behaviour of tumour cells $T$ and immune cells $I$ is presented, where the rate of the effect of vitamins on tumour cells is represented by $c_{1}$. A regular rate of vitamins and maintained rate of blood glucose serve to boost the immune system and its function. Hence, the rate of the effect of vitamins on immune cells is represented by $c_{2}$.

Consequently, TIVM is expressed by the following system:

$$
\begin{aligned}
& \frac{d T}{d t}=\alpha_{1} T\left[1-\alpha_{2} T\right]-\alpha_{3} T I-c_{1} T V, \\
& \frac{d I}{d t}=\sigma-\delta I+\frac{\rho I T}{m+T}-\mu I T+c_{2} I V, \\
& \frac{d V}{d t}=k_{1}-k_{2} V .
\end{aligned}
$$

\section{Analysis of the tumour-immune-vitamins model (TIVM)}

\subsection{Conditions and positivity solution}

TIVM dynamics (10) shows the impact of vitamins intervention on the actions of the immune system by eliminating or suppressing the growth and division of tumour cells. Hence, variables $T(t), I(t)$ and $V(t)$ are real and positive. Furthermore, parameters $\alpha_{1}, \alpha_{2}$, $\alpha_{3}, \sigma, \delta, \rho, m, \mu, c_{1}, c_{2}, k_{1}$ and $k_{2}$ are real, positive and at most equal to one. Therefore, the feasible region is given by

$$
\Omega=\left\{(T, I, V) \in R_{+}^{3}\right\}
$$


We performed simulations using Mathematica program to identify the initial value of vitamins and discovered that the best response occurred when $V(0)=5$. Thus, the initial values of TIVM dynamics (10) are given by

$$
T(0)=1 \quad[26], \quad I(0)=1.22 \quad[20,21] \quad \text { and } \quad V(0)=5 .
$$

For $0 \leq t<\infty$, the set solutions are positive for positive conditions. Based on Theorem 3.1, the following theorem is obtained.

Theorem 5.1 For any time t, the dynamic system region of TIVM $\Omega \subset R_{+}^{3}$ is positivityinvariant, and there exists a positive solution.

Proof Let

$$
\Omega=\Omega_{d}=\left\{(T, I, V) \in R_{+}^{3}, T=\frac{1}{\alpha_{2}}, I=\frac{\sigma}{\delta} \text { and } V=1\right\} .
$$

Thus, for any time $t$, the set solutions $(T(t), I(t), V(t))$ of TIVM (10) are positive. By applying Theorem 3.1, we can prove the solution positivity of $T$ and $I$. First, we illustrate that the solution of third equation of TIVM (10) will be $V(t)=1$. As

$$
\frac{d V}{d t} \leq k_{1}-k_{2} V(t)
$$

by using the separation of variables method, we get

$$
V(t) \leq 1+c e^{-k t}
$$

Let $t \rightarrow \infty$, we get

$$
V(t) \leq 1 .
$$

Since $\Omega=\left\{(T, I, V) \in R_{+}^{3}\right\}$, thus $V(t)=1$ at $t \rightarrow \infty$.

\subsection{Equilibrium points}

The steady states of TIVM in (10) occur as follows:

- $\frac{d T}{d t}=0 \Leftrightarrow$

$$
T\left(\alpha_{1}\left(1-\alpha_{2} T\right)-\alpha_{3} I-c_{1} V\right)=0 .
$$

- $\frac{d I}{d t}=0 \Leftrightarrow$

$$
\begin{aligned}
& \sigma-\delta I+\frac{\rho I T}{m+T}-\mu I T+c_{2} V=0, \\
& \left((\sigma-\delta I)-\mu I T-c_{2} V I\right)(m+T)+\rho I T=0 .
\end{aligned}
$$

- $\frac{d V}{d t}=0 \Leftrightarrow$

$$
V=\frac{k_{1}}{k_{2}} .
$$


Hence, the equilibrium points of TIVM (10) are given as follows:

First, by solving equation (12) for $T$, we get

$$
T=0 \quad \text { or } \quad T=\frac{\alpha_{1}-\alpha_{3} I-c_{1} V}{\alpha_{1} \alpha_{2}} .
$$

Now, substituting $T=0$ into equation (13), we obtain

$$
I=\frac{\sigma k_{2}}{\delta k_{2}-c_{2} k_{1}}, \quad \text { where } \delta>\frac{c_{2} k_{1}}{k_{2}} \text { and } k_{2} \approx 1
$$

Therefore, the first equilibrium point is given by

$$
q_{0}=\left(0, \frac{\sigma k_{2}}{\delta k_{2}-c_{2} k_{1}}, \frac{k_{1}}{k_{2}}\right)
$$

Next, substituting $T=\frac{\alpha_{1}-\alpha_{3} I-c_{1} V}{\alpha_{1} \alpha_{2}}$ into equation (13), we get one real solution, while the other solutions are complex. The real solution is given by

$$
\begin{aligned}
I= & \frac{1}{6 \mu}\left[-\frac{2 A_{2}}{\alpha_{3} k_{2}}+\frac{2^{\frac{4}{3}}\left(A_{2}^{2}-B_{2}\right)}{\left(C_{2}+\sqrt{C_{2}^{2}+4\left(-A_{2}^{2}+B_{2}\right)^{3} \alpha_{3}^{6} k_{2}^{6}}\right)^{\frac{1}{3}}}\right] \\
& +\frac{1}{6 \mu}\left[\frac{2^{\frac{2}{3}}\left(C_{2}+\sqrt{\left.C_{2}^{2}+4\left(-A_{2}^{2}+B_{2}\right)^{3} \alpha_{3}^{6} k_{2}^{6}\right)^{\frac{1}{3}}}\right.}{\alpha_{3}^{2} k_{2}^{2}}\right],
\end{aligned}
$$

where

$$
\begin{aligned}
A_{2}= & \left(\alpha_{1} \alpha_{2} c_{2}+2 \mu c_{1}\right) k_{1}+\left(\alpha_{2} \rho-\left(2+m \alpha_{2}\right)\right), \\
B_{2}= & 3 \mu\left[\mu c_{1}^{2} k_{1}^{2}+\left(\mu+\left(\delta \alpha_{2}+m \mu-\rho\right) \alpha_{2} k_{2}-\left(1+m \alpha_{2}\right) \alpha_{2} c_{2} k_{1}\right) \alpha_{1}^{2} k_{2}\right] \\
& +3 \mu\left[\alpha_{2} c_{2} k_{1}-\left(2+m \alpha_{2}\right) \mu k_{2}+\alpha_{2} \rho k_{2}\right] \alpha_{1} c_{1} k_{1}, \\
C_{2}= & \alpha_{3}^{3} k_{2}^{3}\left[-2 \alpha_{3} \alpha_{2}^{3} c_{2}^{3} k_{1}^{3}+2 \mu^{3} c_{1}^{3} k_{1}^{3}-3 c_{1}^{2} k_{1}^{2} k_{2} \alpha_{1} \mu^{2}\left(\left(2+m \alpha_{2}\right) \mu-\alpha_{2} \rho\right)\right. \\
& -3 c_{1} k_{1} k_{2}^{2} \alpha_{1}^{2} \mu^{2}\left[-2 \mu^{2}+2 \alpha_{2} \mu(-m \mu+\rho)+\alpha_{2}\left(-6 \delta \mu+(-m \mu+\rho)^{2}\right)\right] \\
& +k_{2}^{2} \alpha_{1}^{2}\left[\mu\left(\mu+\alpha_{2}\left(3 \alpha_{2} \delta+m\left(4+m \alpha_{2}\right) \mu\right)\right)+2\left(2+m \alpha_{2}\right) \mu \rho-2 \alpha_{2}^{2} \rho^{2}\right] \\
& +3 c_{1} k_{1} \alpha_{1} \alpha_{2}\left[c_{1}^{2} k_{1}^{2} \mu^{2}-2 c_{1} k_{1} k_{2} \alpha_{1} \mu\left(\mu+2 m \alpha_{2} \mu+\alpha_{2} \rho\right)\right] \\
& \left.+3 c_{2}^{2} k_{1}^{2} \alpha_{1}^{2} \alpha_{2}^{2}\left[c_{1} k_{1} \mu+k_{2} \alpha_{1}\left(\left(m \alpha_{2}-1\right) \mu+2 \alpha_{2} \rho\right)\right]\right] .
\end{aligned}
$$

Furthermore, the second real solution is given by

$$
q_{1}=\left(T^{*}, I^{*}, \frac{k_{1}}{k_{2}}\right)
$$

where

$$
T^{*}=\frac{1}{\alpha_{1} \alpha_{2}}\left[\frac{\alpha_{1} k_{2}-c_{1} k_{1}}{k_{2}}-\alpha_{3} I^{*}\right],
$$




$$
\begin{aligned}
I^{*}= & \frac{1}{6 \mu}\left[-\frac{2 A_{2}}{\alpha_{3} k_{2}}+\frac{2^{\frac{4}{3}}\left(A_{2}^{2}-B_{2}\right)}{\left(C_{2}+\sqrt{C_{2}^{2}+4\left(-A_{2}^{2}+B_{2}\right)^{3} \alpha_{3}^{6} k_{2}^{6}}\right)^{\frac{1}{3}}}\right] \\
& +\frac{1}{6 \mu}\left[\frac{2^{\frac{2}{3}}\left(C_{2}+\sqrt{\left.C_{2}^{2}+4\left(-A_{2}^{2}+B_{2}\right)^{3} \alpha_{3}^{6} k_{2}^{6}\right)^{\frac{1}{3}}}\right.}{\alpha_{3}^{2} k_{2}^{2}}\right] .
\end{aligned}
$$

Remark 5.1 The second solution of equations (12)-(14) is the equilibrium point of TIVM (10) if and only if $0<I<T$. This condition will be satisfied if the immune system is weak or the tumour cells are strengthened, contrary to the case of the response stage. Thus, TIVM has only a response equilibrium point $q_{0}=\left(0, \frac{\sigma k_{2}}{k_{2} \delta-c_{2} k_{1}}, \frac{k_{1}}{k_{2}}\right)$.

\subsection{Stability of tumour-immune-vitamins model (TIVM)}

Applying the concept of the Hartman-Grobman theorem [27], the Jacobian matrix of the nonlinear dynamic system of TIVM (10) is given as follows:

$$
J[T, I, V]=\left[\begin{array}{lll}
F_{T}[T, I, V] & F_{I}[T, I, V] & F_{V}[T, I, V] \\
G_{T}[T, I, V] & G_{I}[T, I, V] & G_{V}[T, I, V] \\
H_{T}[T, I, V] & H_{I}[T, I, V] & H_{V}[T, I, V]
\end{array}\right],
$$

where $F[T, I, V]=\frac{d T}{d t}, G[T, I, V]=\frac{d I}{d t}$ and $H[T, I, V]=\frac{d V}{d t}$. The stability cases of the equilibrium points of TIVM (10) are discussed in what follows.

Theorem 5.2 TIVM (10) has a stable response equilibrium point $q_{0}$.

Proof For analysing the behaviour of $q_{0}$, Jacobian matrix (15) at $q_{0}$ is given as follows:

$$
J[T, I, V]_{q_{0}}=\left[\begin{array}{ccc}
\frac{\alpha_{1} k_{2}-c_{1} k_{1}}{k_{2}}+\frac{\alpha_{3} \sigma k_{2}}{c_{2} k_{1}-\delta k_{2}} & 0 & 0 \\
\frac{(-m \mu \mu \rho) \sigma 2}{\left(c_{2} k_{1}+\delta k_{2}\right) m} & \frac{c_{2} k_{1}-\delta k_{2}}{k_{2}} & \frac{c_{2} k_{2} \sigma}{c_{2} k_{2}+\delta k_{2}} \\
0 & 0 & -k_{2}
\end{array}\right] .
$$

To analyse the behaviour of the equilibrium point $q_{0}$, the characteristic equation of matrix (16) is computed as follows:

$$
\left(-k_{2}-\lambda\right)\left(\frac{c_{2} k_{1}-\delta k_{2}}{k_{2}}-\lambda\right)\left(\frac{\alpha_{1} k_{2}-c_{1} k_{1}}{k_{2}}-\frac{\alpha_{3} \sigma k_{2}}{c_{2} k_{1}+\delta k_{2}}-\lambda\right)=0 .
$$

Hence, the eigenvalues of matrix (16) are given as follows:

$$
\begin{aligned}
\lambda_{1} & =-k_{2}, \\
\lambda_{2} & =\frac{c_{2} k_{1}-\delta k_{2}}{k_{2}}<0, \quad \text { where from the equilibrium point } q_{1}, \delta>\frac{c_{2} k_{1}}{k_{2}} \equiv \delta_{1}, \\
\lambda_{3} & =\frac{\alpha_{1} k_{2}-c_{1} k_{1}}{k_{2}}-\frac{\alpha_{3} \sigma k_{2}}{\delta-\frac{c_{2} k_{1}}{k_{2}}} \\
& =\frac{\left(\alpha_{1}-\frac{c_{1} k_{1}}{k_{2}}\right)\left(\delta-\delta_{1}\right)-\alpha_{3} \sigma k_{2}^{2}}{\left(\delta-\delta_{1}\right) k_{2}} .
\end{aligned}
$$


Then TIVM will be stable if $\lambda_{3}<0$, which can be shown as follows:

$$
\begin{aligned}
& \left(\alpha_{1}-\frac{c_{1} k_{1}}{k_{2}}\right)\left(\delta-\delta_{1}\right)-\alpha_{3} \sigma k_{2}^{2}<0, \\
& \alpha_{1}-\frac{c_{1} k_{1}}{k_{2}}<\frac{\alpha_{3} \sigma k_{2}^{2}}{\delta-\delta_{1}}, \\
& \alpha_{1}<\frac{\alpha_{3} \sigma k_{2}^{2}}{\delta-\delta_{1}}+\frac{c_{1} k_{1}}{k_{2}} .
\end{aligned}
$$

Hence, the equilibrium point $q_{0}$ is stable if and only if

$$
0<\alpha_{1}<\frac{\alpha_{3} \sigma k_{2}^{2}}{\delta-\delta_{1}}+\frac{c_{1} k_{1}}{k_{2}}
$$

Remark 5.2 Comparing the response stage equilibrium points of TIUNHDM and TIVM, we make the following remarks:

- The stability of the TIVM response stage results from vitamins intervention. This indicates that vitamins contributed to boosting the immune system and regulating the behaviour of the solutions around the equilibrium point $q_{0}$. Compared to the initial value of the immune cells, this effect can be deduced from the increase in immune cells at this stage. This increase was not observed in the response stage of TIUNHDM, where the response of the immune cells was represented by the initial value problem.

- Tumour cells exist to survive and do not have an automatic death mechanism, compared to other types of cells. On the other hand, there are internal factors that contribute to their growth suppression. The growth of tumour cells may be delayed by boosting and increasing the immune cells. Dynamically, this is only possible if and only if the suppression rate of tumour cells by immune cells is higher than the growth rate of tumour cells; that is, $\alpha_{3}<\alpha_{1}$.

- The parametric solution of TIVM and its behaviour around the equilibrium point are shown in Figs. 3-4.

Figure 3 Parametric solution of TIVM

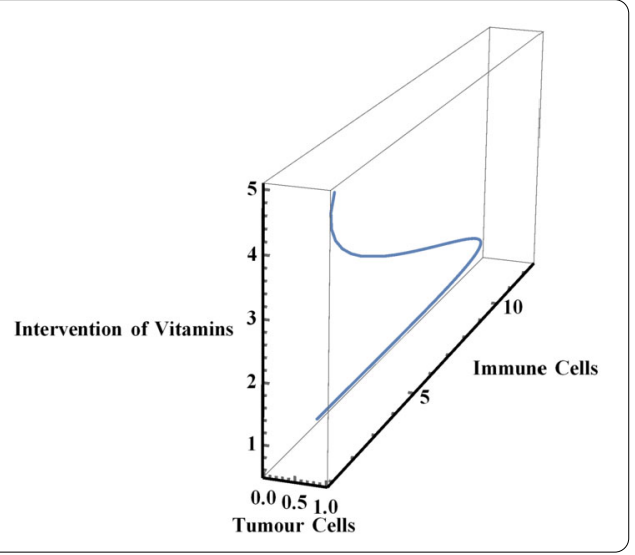




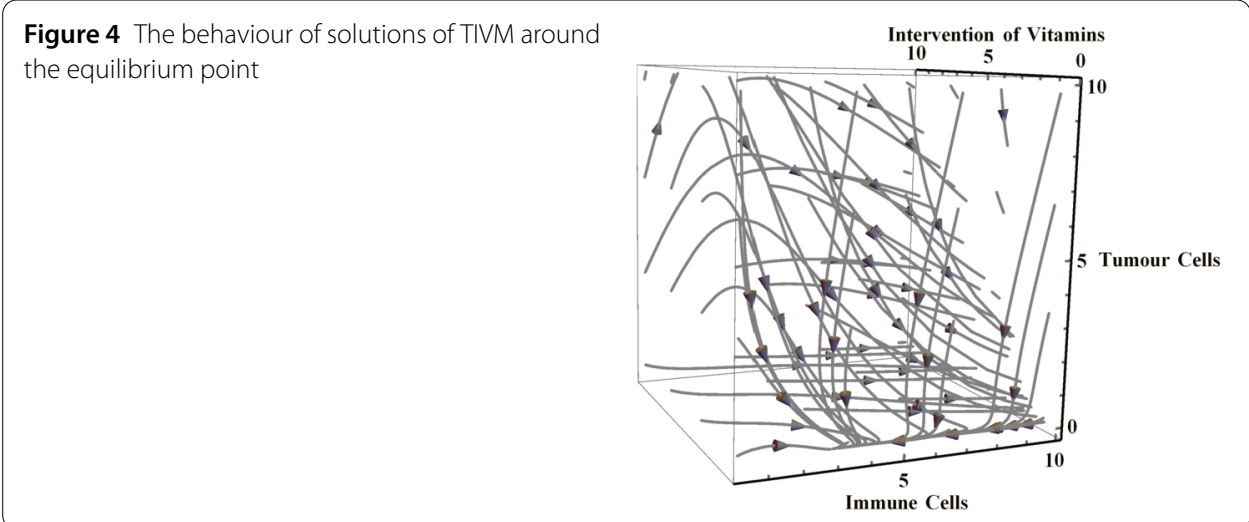

\section{Numerical simulation}

NDSolve command in Mathematica 11.2 is used for the simulations of TIUNHDM (1) and TIVM (10). A fourth order Runge-Kutta method is used via NDSolve to obtain a stable and convergent solution for the model simulations. The simulations of TIUNHDM and TIVM were carried out by selecting step integration as $\frac{1}{1000}$ and thirty days as a time unit. These simulations showed that the shifting from an unhealthy to a healthy diet had a substantial impact on suppressing tumour cells growth. Additionally, the vitamins which came from a natural source of foods and beverages in a healthy diet played a role in boosting the immune system and improving its functionality in suppressing or delaying tumour cells growth, especially in primary stages. As illustrated in Figs. 5-8, the residual error values validated the robustness of the proposed methods. Both TIUNHDM (1) and TIVM (10) simulation results were compared with numerical results evaluating estrogen effect on the breast cancer dynamics, as presented in [11].

The simulation results of both TIUNHDM and TIVM showed that the appearance of tumour cells stimulated the division of immune cells, even when the immune system was weak, but there was a differentiation in immune cell functions. This differentiation could be deduced from the values of parameters that affected the behaviour of both TIUNHDM and TIVM. From the simulation of both models, it can be concluded that there are two parameters that directly affect the behaviour of tumour and immune cells, namely the suppression rate of the tumour cells by immune cells denoted by $\alpha_{3}$ and the suppression rate of immune cells as a result of being attacked by tumour cells denoted by the parameter $\mu$. Furthermore, it is obvious that delaying the growth of tumour cells occurred if the following conditions are satisfied:

The suppression rate of tumour cells $>$ The suppression rate of immune cells, $\alpha_{3}>\mu$.

Vitamins were used to regulate the rates of parameters $\alpha_{3}$ and $\mu$ and the TIVM simulation results showed that the body intake needed $k_{1}=55 \%$ from vitamins derived from natural foods and beverages, where the highest rate of vitamins will support attack on the tumour cells and other rates will support the immune cells as follows: the rate involving vitamins was simulated by $k_{2}=97 \%, c_{1}=60 \%$ and $c_{2}=36 \%$. The cell behaviour in TIUNHDM and TIVM is shown in Figs. 9-10. 


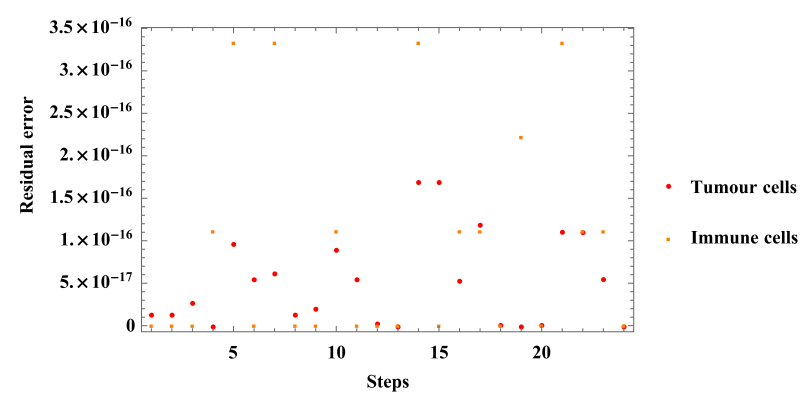

Figure 5 Residual error at various steps for TIUNHDM

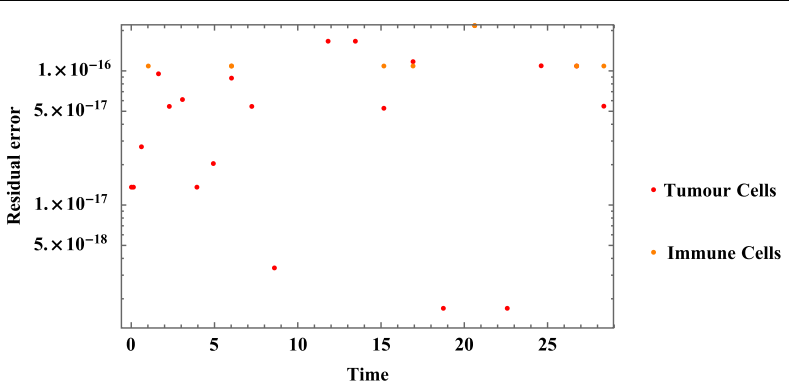

Figure 6 Residual error at time $t$ for TIUNHDM

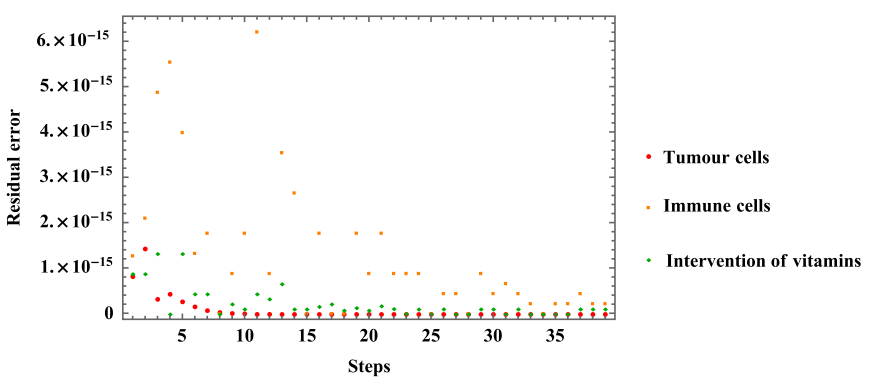

Figure 7 Residual error at various steps for TIVM

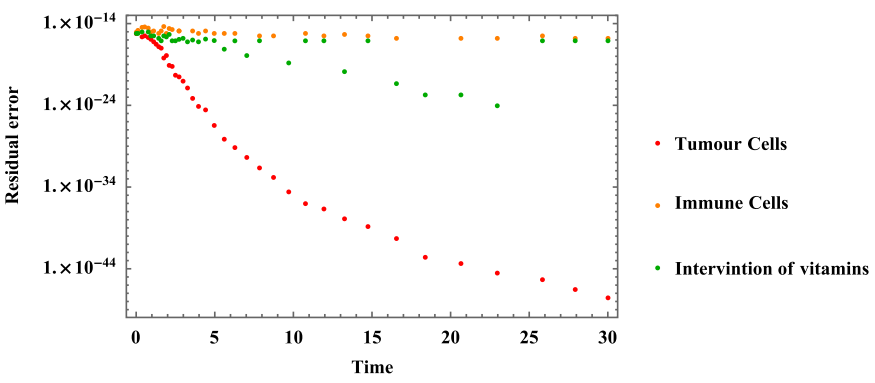

Figure 8 Residual error at time $t$ for TIVM 


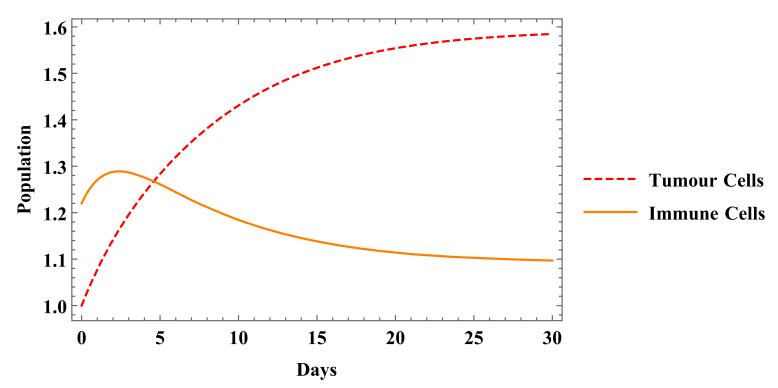

Figure 9 The cell behaviour in TIUNHDM, with $\alpha_{1}=0.4426, \alpha_{2}=0.4, \alpha_{3}=0.1469, \sigma=0.7, \delta=0.57$, $\mu=0.3634, \rho=0.7829$ and $m=0.8620$

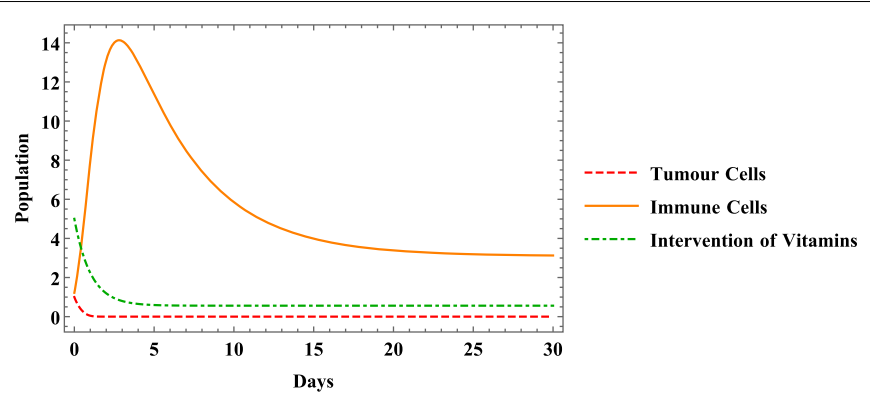

Figure 10 The cell behaviour in TIVM, with $\alpha_{1}=0.4426, \alpha_{2}=0.4, \alpha_{3}=0.5140, c_{1}=0.6142, \sigma=0.7, \delta=0.57$ $\mu=0.1859, \rho=0.7829, m=0.8620, c_{2}=0.3628, k_{1}=0.5463$ and $k_{2}=0.9757$

\section{Conclusion}

The appearance of tumour cells in tissues is caused by the failure of the immune system to eliminate or suppress abnormal cells, as was dynamically shown in [20]. In this paper, a mathematical tumour-immune-unhealthy diet model (TIUNHDM) and a tumourimmune-vitamins model (TIVM), which are governed by ordinary differential equations, were developed to illustrate the existence of relationship between diet habits and the growth of tumour cells. The analysis of TIUNHDM showed that the immune system became involved in the interaction as a result of the activity of the tumour cells. It is possible to turn these immune cells into cancer immune cells. Mathematically, there was no recovery stage for TIUNHDM since the immune cells did not go to zero. Moreover, there was no co-existent stage between the cells, where there was no consideration of normal cells. From the results of simulation of the model, it can be concluded that the model achieved cells interaction when the suppression rate of the immune cells is greater than the suppression rate of the tumour cells. Additionally, we suggested that status of the immune system is boosted by shifting from an unhealthy diet to a healthy diet that is rich in vitamins. This effect was incorporated into TIUNHDM to form the tumour-immune-vitamins model (TIVM). It can be concluded from the analysis and simulation of TIVM that boosting the immune cells by the vitamins intervention may serve to delay the growth and division of tumour cells. Dynamically, TIVM does not have an interaction stage, which indicates that there was an opposite relationship between boosting the immune system by the vitamins intervention and delaying the growth and division of tumour cells. According to the simulation of TIVM, body intake of meals containing 55\% from vitamins per day is recommended to boost the immune system and prevent tumour cells from developing. In 
summary, this paper evaluated the effects of vitamins intervention on delaying tumour cell growth and reducing the risk of cancer through dynamic modelling. We hope to conduct more experimental studies to clinically investigate the results of this paper and contribute to identifying the specific diet patterns which are most suitable for reducing the growth of tumour cells. In future studies, we will develop these models by studying the effects of other factors which are correlated with an increase in the risk of cancer based on the effects of diet and the dynamics of other diseases.

\section{Acknowledgements}

We thank Universiti Kebangsaan Malaysia for providing financial support and facilities for this research under the grant GUP-2017-112.

Funding

This study is supported by Universiti Kebangsaan Malaysia (The grant is GUP-2017-112).

Availability of data and materials

The data used to support the findings of this study are included in the article.

\section{Competing interests}

The authors declare that they have no competing interests.

\section{Authors' contributions}

All authors contributed equally to this manuscript. All authors read and approved the final manuscript.

\section{Author details}

'Department of Mathematics \& Statistics, College of Science, Taibah University, 41911, Yanbu, Almadinah Almunawarah, Saudi Arabia. ${ }^{2}$ Department of Mathematical Sciences, Faculty of Science \& Technology, Universiti Kebangsaan Malaysia, 43600, Bangi, Selangor, Malaysia.

\section{Publisher's Note}

Springer Nature remains neutral with regard to jurisdictional claims in published maps and institutional affiliations.

Received: 4 June 2020 Accepted: 28 July 2020 Published online: 08 August 2020

\section{References}

1. Alaini, R., Rajikan, R., Elias, S.M.: Diet optimization using linear programming to develop low cost cancer prevention food plan for selected adults in Kuala Lumpur, Malaysia. BMC Public Health 19(4), 546 (2019)

2. Rudolph, A., Chang-Claude, J., Schmidt, M.K.: Gene-environment interaction and risk of breast cancer. Br. J. Cancer 114(2), 125 (2016)

3. Rawla, P., Sunkara, T., Gaduputi, V.: Epidemiology of pancreatic cancer: global trends, etiology and risk factors. World J. Oncol. 10(1), 10 (2019)

4. Anand, P., Kunnumakara, A.B., Sundaram, C., Harikumar, K.B., Tharakan, S.T., Lai, O.S., Sung, B., Aggarwal, B.B.: Cancer is a preventable disease that requires major lifestyle changes. Pharm. Res. 25(9), 2097-2116 (2008)

5. Khan, H., Hussain, F.H., Samad, A.: Cure and prevention of diseases with vitamin C into perspective: an overview. J. Crit. Rev. 7(4), 289-293 (2019)

6. Jafari, D., Esmaeilzadeh, A., Mohammadi-Kordkhayli, M., Rezaei, N.: Vitamin C and the immune system. In: Nutrition and Immunity. Springer, Cham (2019)

7. Hong, Y.-J., Kim, J., Lee, H.Y., Rim, C.H.: Development of the anti-cancer food scoring system 2.0: validation and nutritional analyses of quantitative anti-cancer food scoring model. Nutr. Res. Pract. 14(1), 32-44 (2020)

8. Kuznetsov, V.A., Makalkin, I.A., Taylor, M.A., Perelson, A.S.: Nonlinear dynamics of immunogenic tumors: parameter estimation and global bifurcation analysis. Bull. Math. Biol. 56(2), 295-321 (1994)

9. Kirschner, D., Panetta, J.C.: Modeling immunotherapy of the tumor-immune interaction. J. Math. Biol. 37(3), 235-252 (1998)

10. Mayer, H., Zaenker, K., An Der Heiden, U.: A basic mathematical model of the immune response. Chaos, Interdiscip. J. Nonlinear Sci. 5(1), 155-161 (1995)

11. Mufudza, C., Sorofa, W., Chiyaka, E.T.: Assessing the effects of estrogen on the dynamics of breast cancer. Comput. Math. Methods Med. 2012, 473572 (2012)

12. Glick, A., Mastroberardino, A.: An optimal control approach for the treatment of solid tumors with angiogenesis inhibitors. Mathematics 5(4), 49 (2017)

13. Alqudah, M.A.: Cancer treatment by stem cells and chemotherapy as a mathematical model with numerical simulations. Alex. Eng. J. (2020)

14. Simbawa, E:: Mechanistic model for cancer growth and response to chemotherapy. Comput. Math. Methods Med. 2017, 3676295 (2017)

15. Villasana, M., Radunskaya, A.: A delay differential equation model for tumor growth. J. Math. Biol. 47(3), 270-294 (2003) 
16. Khajanchi, S., Perc, M., Ghosh, D.: The influence of time delay in a chaotic cancer model. Chaos, Interdiscip. J. Nonlinear Sci. 28(10), 103101 (2018)

17. Elaiw, A., Al Agha, A.: Analysis of a delayed and diffusive oncolytic $m 1$ virotherapy model with immune response. Nonlinear Anal., Real World Appl. 55, 103116 (2020)

18. Ku-Carrillo, R.A., Delgadillo, S.E., Chen-Charpentier, B.: A mathematical model for the effect of obesity on cancer growth and on the immune system response. Appl. Math. Model. 40(7-8), 4908-4920 (2016)

19. Ku-Carrillo, R.A., Delgadillo-Aleman, S.E., Chen-Charpentier, B.M.: Effects of the obesity on optimal control schedules of chemotherapy on a cancerous tumor. J. Comput. Appl. Math. 309, 603-610 (2017)

20. Alharbi, S.A., Rambely, A.S.: A dynamic simulation of the immune system response to inhibit and eliminate abnormal cells. Symmetry 11(4), $572(2019)$

21. Alharbi, S.A., Rambely, A.S., Almatroud, A.O.: Dynamic modelling of boosting the immune system and its functions by vitamins intervention. Nonlinear Dyn. Syst. Theory 19(2), 1-11 (2019)

22. Yusof, A.S., Isa, Z.M., Shah, S.A.: Dietary patterns and risk of colorectal cancer: a systematic review of cohort studies (2000-2011). Asian Pac. J. Cancer Prev. 13(9), 4713-4717 (2012)

23. Marwitz, S.E., Woodie, L.N., Blythe, S.N.: Western-style diet induces insulin insensitivity and hyperactivity in adolescent male rats. Physiol. Behav. 151, 147-154 (2015)

24. Sample, C.H., Martin, A.A., Jones, S., Hargrave, S.L., Davidson, T.L.: Western-style diet impairs stimulus control by food deprivation state cues: implications for obesogenic environments. Appetite 93, 13-23 (2015)

25. Abernathy, K., Abernathy, Z., Baxter, A., Stevens, M.: Global dynamics of a breast cancer competition model. Differ. Equ. Dyn. Syst. 1-15 (2017)

26. Alharbi, S.A., Rambely, A.S.: Dynamic simulation for analyzing the effects of the intervention of vitamins on delaying the growth of tumor cells. IEEE Access 7, 128816-128827 (2019)

27. Hartman, P.: Ordinary Differential Equations. Wiley, New York (1964)

28. World Cancer Research Fund/American Institute for Cancer Research: Diet, nutrition, physical activity and cancer: a global perspective Continuous Update Project Expert Report (2018)

29. Rondanelli, M., Faliva, M.A., Miccono, A., Naso, M., Nichetti, M., Riva, A., Guerriero, F., De Gregori, M., Peroni, G., Perna, S. Food pyramid for subjects with chronic pain: foods and dietary constituents as anti-inflammatory and antioxidant agents. Nutr. Res. Rev. 31(1), 131-151 (2018)

30. Marie, S.K.N., Shinjo, S.M.O.: Metabolism and brain cancer. Clinics 66, 33-43 (2011)

\section{Submit your manuscript to a SpringerOpen ${ }^{\circ}$ journal and benefit from:}

- Convenient online submission

Rigorous peer review

Open access: articles freely available online

- High visibility within the field

- Retaining the copyright to your article

Submit your next manuscript at $\gg$ springeropen.com 